\title{
Zonisamide Treatment Delays Motor Neuron Degeneration and Astrocyte Proliferation in Wobbler Mice
}

\author{
Takehisa Hirayama ${ }^{a}$, Yasushiro Yoshii ${ }^{a}$, Kiyokazu Kawabe ${ }^{a}$, Ken Ikeda ${ }^{a}$, b
}

\begin{abstract}
Background: Zonisamide (ZNS) had multifunctional effects on several kinds of neurons. Little is known about neuroprotective effects of ZNS on motor neurons. We aimed to study whether this drug can attenuate motor neuron degeneration in wobbler mice.

Methods: Wobbler mice were injected daily two doses of ZNS (0.2 $\mathrm{mg} / \mathrm{kg}, 2.0 \mathrm{mg} / \mathrm{kg}$, i.p.) or vehicle from aged $3-4$ weeks at disease onset for more than 4 weeks. Motor function was evaluated by pullstrength and deformity scale of the forelimbs. Those symptomatic assessment and body weight were measured weekly. Neuropathological changes of the biceps muscle and the cervical cord were analyzed at 4 weeks posttreatment.
\end{abstract}

Results: ZNS treatment $(2.0 \mathrm{mg} / \mathrm{kg})$ significantly delayed progression of forelimb motor dysfunction compared to vehicle $(\mathrm{P}<$ $0.01)$. Gain of body weight did not differ statistically between three groups. Higher doses of ZNS administration decreased denervation atrophy in the biceps muscle $(\mathrm{P}<0.01)$, suppressed loss of motor neurons $(\mathrm{P}<0.01)$ and inhibited astrocyte proliferation $(\mathrm{P}<0.01)$.

Conclusions: The present study indicated that ZNS treatment attenuated motor neuron degeneration and astrocytosis in the wobbler mouse. This drug may have a therapeutic potential for motor neuron disease.

Keywords: Zonisamide; Neuroprotective effect; Wobbler mouse; Motor dysfunction; Motor neuron degeneration; Astrocyte proliferation

Manuscript accepted for publication October 17, 2011

${ }^{\text {a } D e p a r t m e n t ~ o f ~ N e u r o l o g y, ~ T o h o ~ U n i v e r s i t y ~ O m o r i ~ M e d i c a l ~ C e n t e r, ~}$ 6-11-1, Omorinishi, Otaku, Tokyo, 143-8541, Japan

${ }^{\mathrm{b} C}$ Corresponding author: Ken Ikeda. Email: keni@med.toho-u.ac.jp

doi:10.4021/jnr59w

\section{Introduction}

Zonisamide (ZNS), 1, 2-benzisoxazole-3-methanesulfonamide is used widely as an anticonvulsant drug. Anti-epileptic mechanisms revealed blockage of voltage-gated sodium channels $[1,2]$ and T-type calcium channels [3]. In addition to the anti-convulsant properties, ZNS reduced neonatal hypoxic-ischemic damage in experimental animals [4]. This ant-epileptic drug prevented against dopamine quinone formation induced by excess amount of cytosolic dopamine outside the synaptic vesicles [5] and also attenuated loss of dopaminergic neurons after 1-methyl-4-phenyl-1, 2, 3, 6-tetrahydropyridine (MPTP)-treated mice [6] Therefore, ZNS had multifunctional effects on several kinds of neurons [7]. However, little is known about a neuroprotective potential of ZNS on damaged motor neurons. The wobbler mouse is an animal model of lower motor neuron degeneration $[8$, 9]. Here we aimed to evaluate whether this drug can delay symptomatic and neuropathological deterioration in wobbler mice.

\section{Materials and Methods}

\section{Animals}

Wobbler mice (wr/wr) and their normal littermates (NFR/wr) in the present study were derived from Department of Neurology, Toho University School of Medicine, Omori mouse colony, Japan. Mice were treated according to the guidelines of the Toho University Animal Committee. This study was approved by the ethics committee of Toho University School of Medicine.

\section{ZNS administration}

Purified ZNS was used in this study and provided by Dainippon Sumitomo Pharma Co., Ltd, Osaka, Japan. Wobbler mouse initially developed a trembling body at the age of $3-4$ weeks. This shaking body was diagnosed as clinical onset of disease. Immediately after symptomatic onset, affected mice 


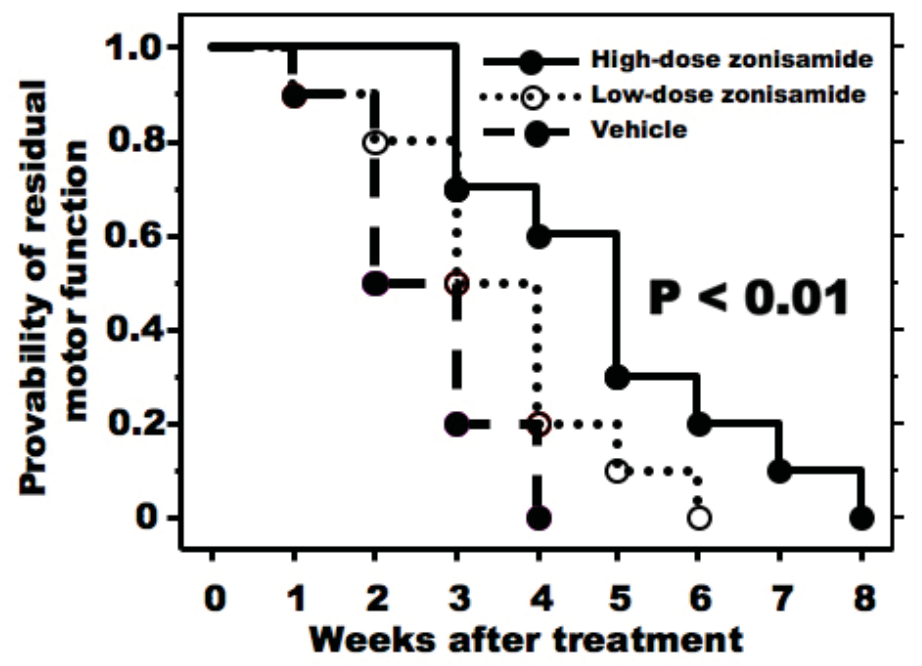

Figure 1. Progression of forelimb motor deficits. High-dose zonisamide administration retards progression of forelimb dysfunction significantly. $(\mathrm{P}<0.01$ by Kaplan-Meyer method)

were administered daily two doses of ZNS $(0.2 \mathrm{mg} / \mathrm{kg} /$ day or $2.0 \mathrm{mg} / \mathrm{kg} / \mathrm{day}$, i.p.) or vehicle from aged 3 - 4 weeks in a blind fashion. The treatment persisted until the terminal stage of forelimb motor dysfunction for more than 4 weeks ( $n=10$ /group). In another neuropathological experiment, the treatment was performed from aged $3-4$ weeks to $7-8$ weeks for four weeks ( $\mathrm{n}=10$ /group).

\section{Symptomatic assessment}

Forelimb function was assessed by forelimb deformity scale and pull-strength of the forelimbs. Deformity scale was graded at1, paw atrophy; 2, curled digits; 3, curled wrists; and 4, forelimb J-shaped flexion to the chest. Pull-strength of the forelimbs was measured by holding a tail in the air, and grasping a horizontal wire gauge connected to a special dynamometer with both paws. The maximum strength was determined. Absence of forelimb motor function was defined by deformity scale of grade 3 or 4 in both forelimbs. When this motor function was abolished, pull-strength of forelimbs was set at 0 gram. These symptomatic assessments and the measurement of body weight were performed weekly from the clinical onset to the age of $7-8$ weeks at 4 weeks after treatment ( $\mathrm{n}=10$ / group).

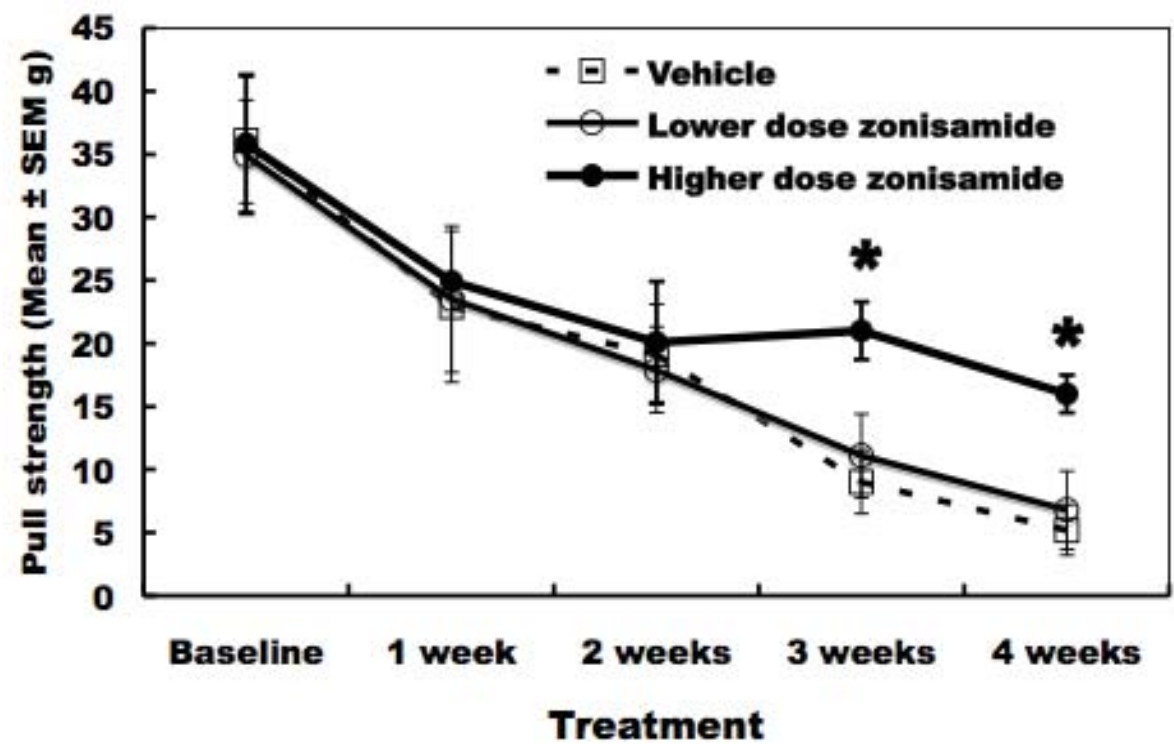

Figure 2. Pull-strength of the forelimbs. High-dose zonisamide administration delays decline of pull-strength at 3 and 4 weeks after treatment. ( ${ }^{\star} P<0.01$ at 3 and 4 weeks after treatment by unpaired Student's t-test) 
B

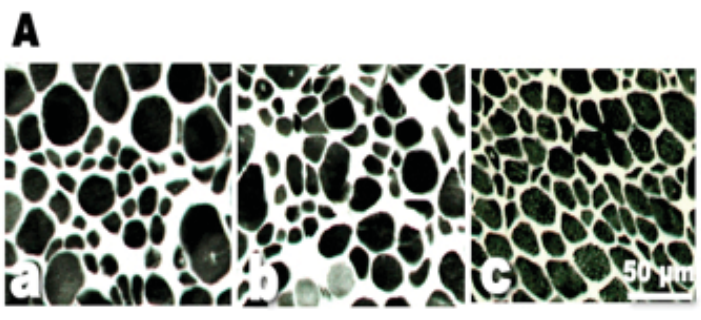

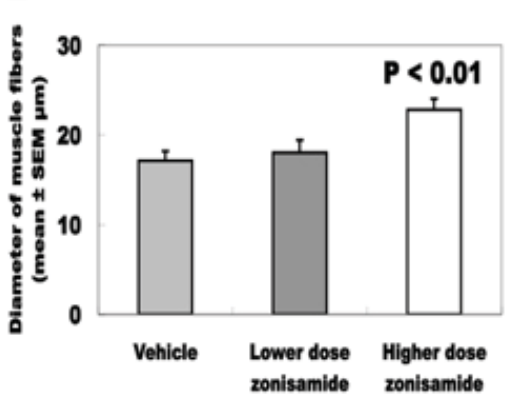

Figure 3. Denervation changes of the biceps muscles. (A) ATPase staining of biceps muscles. (a) Vehicle treatment. (b) Low-dose zonisamide treatment. (c) High-dose zonisamide treatment. (B) Treatment with high-dose zonisamide increases the mean diameter of muscle fibers at $30 \%$. $(P<0.01$ between the high-dose zonisamide group and the vehicle group by one-way ANOVA followed by Scheffe's test)

\section{Morphometry of biceps muscles}

Under intraperitoneal administration of pentobarbital (40 $\mathrm{mg} / \mathrm{kg}$ ), the right biceps muscles were removed under a dissecting microscope at post-treatment 4 weeks. The muscles were weighed and frozen. Serial $10 \mu \mathrm{m}$ sections were stained with routine ATPase. Two areas from the short and long heads of biceps muscle were selected arbitrarily for histometry. Two hundred muscle fibers per mouse were analyzed, and the mean diameter of muscle fibers was calculated in three groups of wobbler mice treated with two doses of zonisamide or vehicle ( $\mathrm{n}=10$ /group).

\section{Number of spinal motor neurons and astrocytes}

After dissection of the biceps muscle, wobbler mice were perfused through an intracardiac catheter with phosphate buffered saline followed by $4 \%$ paraformaldehyde / $1 \%$ glutaraldehyde / 0.1 M sodium phosphate buffer, $\mathrm{pH}$ 7.4. Laminectomy was performed and the cervical spinal cord was removed under a dissecting microscope. The C5-6 cord was taken for analysis for motor neurons and astrocytes. The spinal cord segments were embedded in paraffin, sectioned serially at $8 \mu \mathrm{m}$ in the transverse plane, and stained with cresyl-violet. At every fifth section, large motor neurons (larger than $20 \mu \mathrm{m}$ ) with prominent nucleoli were counted in the ventral portion of the C5-6 cord. The total number of large motor neurons was counted in zonisamide- and vehicle-treated wobbler mice. For immunohistochemistry of glial fibrillary acidic protein (GFAP) to identify astrocytes, paraffin-embedded sections as described above were deparaffinized and incubated overnight at $4{ }^{\circ} \mathrm{C}$ with rabbit antibody to GFAP (Dako, Denmark) at a dilution of 1:1000. GFAP-immunoreactive astrocytes were investigated with a light microscope at magnification X 200. The number of astrocytes (cells $/ \mathrm{mm}^{2}$ ) was measured in the ventral horn of the C5-6 cord, using a computer-associated image analyzer software (MacSCOPE, Mitani Corporation, Fukui, Japan).

\section{Statistical analyses}

All data were expressed as mean \pm SEM. Progression of forelimb deformity was analyzed by Kaplan-Meyer method. Statistical analysis for pull-strength of the forelimbs used unpaired Student's t-test. Statistical comparison of the number of motor neurons and astrocytes was performed by oneway analysis of variance (ANOVA) followed by the post hoc Scheffe's test. The significance level was set at 0.05 in all statistical tests.

\section{Results}

\section{Forelimb motor function and body weight}

As compared to vehicle-treated wobbler mice, higher dose of ZNS administration delayed the progression of forelimb deformity (Fig. 1). Pull-strength of the forelimbs was decreased gradually in vehicle-treated mice. ZNS-treated mice $(2.0 \mathrm{mg} / \mathrm{kg})$ significantly slowed decline of pull-strength from 3 weeks after treatment compared to vehicle-treated mice (Fig. 2). High dose of ZNS-treated mice had approximate $2-5 \%$ greater gain of body weight compared to mice treated with vehicle and low dose of ZNS. Body weight did not differ statistically between three groups at 4 weeks posttreatment (the age of 7 - 8 weeks).

\section{Degree of muscle atrophy}

The changes of the biceps muscle are expressed in Figure 3. The biceps muscle weight was increased approximately $30 \%$ in high dose of ZNS-treated wobbler mice $(5.9 \pm 0.2 \mathrm{mg})$ compared to mice treated with low dose of ZNS $(4.6 \pm 0.3)$ and vehicle $(4.5 \pm 0.4)$. The mean diameter of muscle fibers 
A

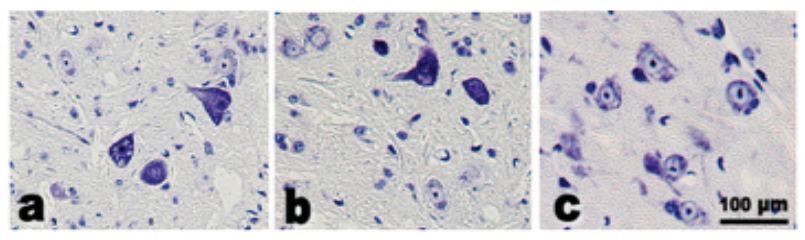

B

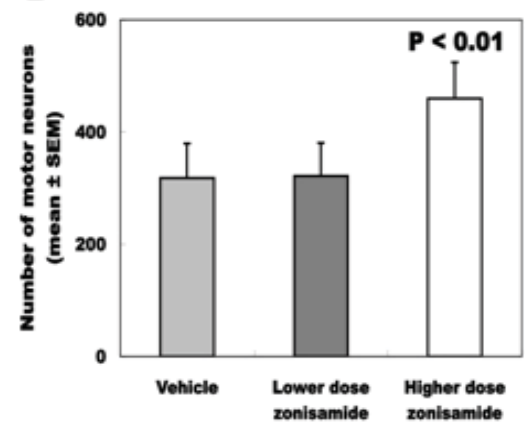

Figure 4. Total number of survived motor neurons. (A) Nissl staining of C5-6 cord. (a) Vehicle treatment. (b) Low-dose zonisamide treatment. (c) High-dose zonisamide treatment. (B) High-dose zonisamide administration inhibits the loss of motor neurons at 45\%. ( $P<0.01$ between the high-dose zonisamide group and the vehicle group by one-way ANOVA followed by Scheffe's test)

was also increased significantly in the high-dose ZNS group $(22.8 \pm 1.2 \mu \mathrm{m})$ compared to the low-dose ZNS group (18.0 $\pm 1.4)$ and the vehicle group $(17.1 \pm 1.1)$.

\section{Survival of spinal motor neurons}

The total number of spinal motor neurons differed statistically between wobbler mice treated with higher dose of ZNS $(459.7 \pm 64.0)$, lower dose $(321.6 \pm 58.8)$ and vehicle (317.6 \pm 61.8). High-dose ZNS administration inhibited the loss of motor neurons approximately $45 \%$ in wobbler mice (Fig. 4).

\section{Astrocyte proliferation}

The number of GFAP-immunoreactive astrocytes was $9.5 \pm$ 3.9 cells $/ \mathrm{mm}^{2}$ in higher dose of ZNS, $19.8 \pm 5.3$ in lower dose of ZNS and $23.7 \pm 4.0$ in vehicle. Higher dose of ZNS significantly decreased astrocyte proliferation approximately $60 \%$ (Fig. 5).

\section{Discussion}

The wobbler mouse exhibits autosomal recessive mutation of vacuolar-vesicular protein sorting (VPS) 54 on mouse chromosome 11. Missense mutation of VPS54 causes spinal motor neuron degeneration and deficit of spermiogenesis in this animal [9]. The symptomatic onset is a shaking body at the age of 3 - 4 weeks. Muscle weakness and contracture in the forelimbs progress rapidly until 8 weeks of age. Afterwards, these neuromuscular deficits progressed slowly. Motor function of the forelimbs declines to the terminal stage at 8 - 10 weeks of age. The survival of affected mice is approximately 1 year when they are maintained together with normal littermates. Therefore, we evaluated forelimb motor function from clinical onset to the age of 7 - 8 weeks.

The neuropathological features reveal degeneration of spinal motor neurons and proximal axonopathy predominantly in the cervical cord, leading to denervation muscle atrophy in the forelimbs. Although the wobbler mouse is not an animal model of amyotrophic lateral sclerosis (ALS),
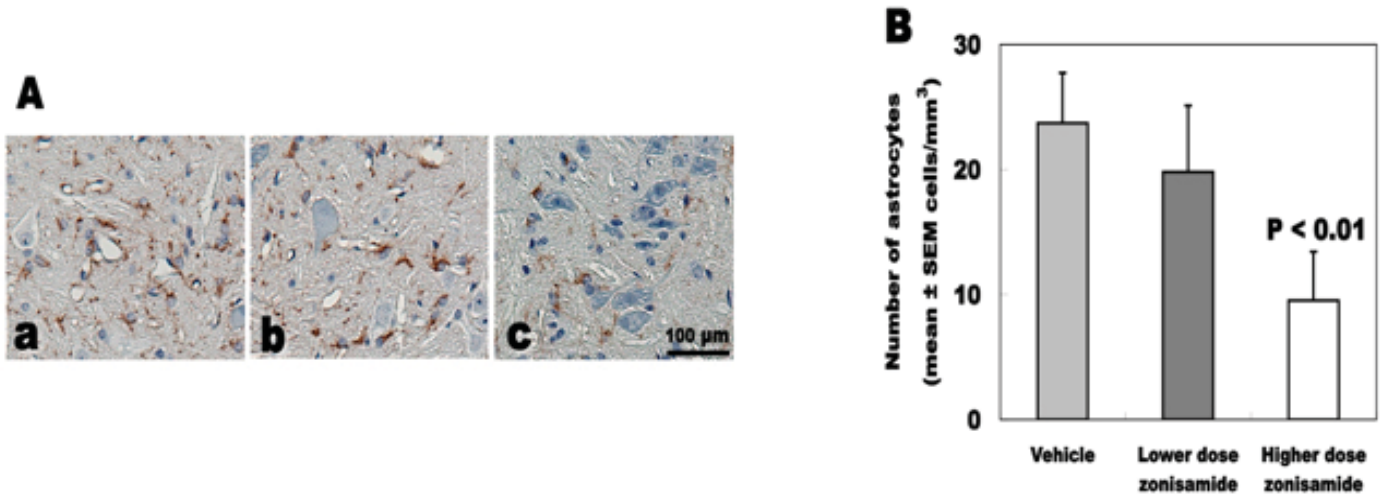

Figure 5. Number of GFAP-immunoreactive astrocytes. (A) GFAP immunostaining of C5-6 cord. (a) Vehicle treatment. (b) Low-dose zonisamide treatment. (c) High-dose zonisamide treatment. (B) High-dose zonisamide treatment decreases degree of astrocyte proliferation at $60 \%$. $(P<0.01$ between the high-dose zonisamide and the vehicle group by oneway ANOVA followed by Scheffe's test) 
the constant symptomatic and neuropathological hallmarks have benefits for therapeutic model of lower motor neuron degeneration [10-13]. Moreover, a recent study has highlighted Tar-DNA binding protein-43 (TDP-43) and ubiquitin changes of the spinal cord in this animal that were characterized in sporadic ALS patients [14]. In the present study, ZNS treatment $(2.0 \mathrm{mg} / \mathrm{kg})$ prolonged forelimb motor function in wobbler mice. The neuropathological analyses suggested that this drug attenuated denervation muscle atrophy, motor neuron degeneration and astrocyte proliferation.

ZNS was reported to have benefits in several experimental animal models [7], including epileptic seizure [15, 16], brain ischemia [4, 17] and Parkinson's disease [6, 18, 19]. Intraperitoneal administration of ZNS $(40 \mathrm{mg} / \mathrm{kg} /$ day for 7 days) protected dopaminergic neurons in the substantia nigra of MPTP-mice, and also inhibited proliferation of GFAPimmunoreactive astrocytes and microglia in the substantia nigra [6]. This drug rescued dopaminergic neurons and enhanced the number of S100b-immunoreactive astrocytes in 6-hydroxydopamine-injected hemiparkinsonian mice [18]. ZNS-treated MPTP-mice (40 mg/kg/day for 1 day after final administration of MPTP) recovered dopaminergic neurons and potentiated proliferation of S100- and GFAP-immunoreactive astrocytes in the substantia nigra [19]. With respect to astrocytic changes in wobbler mice, astrocytosis was suggested to occur before the loss of motor neurons in wobbler mice [20]. Spinal cord GFAP levels were increased in wobbler mice at aged 2 months. Immunohistochemical studies suggested astrocyte overgrowth in the cervical cord of wobbler mice at the age of 2-3 months [21, 22]. Astrocytes derived from wobbler murine neural precursor cells displayed impairment of glutamate homeostasis [23]. These previous studies advocated that interaction between motor neurons and astrocytes could play a crucial role in the pathogenesis of wobbler mouse motor neuron degeneration. In the present study, ZNS treatment saved degenerating motor neurons and suppressed astrocytosis in wobbler mice. These results supported that both direct effects on motor neurons and astrocyte-dependent effects might contribute to the neuroprotective mechanism of ZNS in this animal.

In gerbil ischemic brain injury, ZNS decreased ischemiainduced extracellular glutamate accumulation in the hippocampus [17]. Excesses of glutamate activates N-methyl-Daspartate receptors in the neurons, and can induce production of free radicals and nitric oxide (NO), leading to neuronal death. ZNS decreased production of reactive oxygen species in cultured hippocampal neurons exposed by kainic acid [24]. This drug scavenged hydroxyl and NO radicals in electron spin resonance study [25]. We reported previously that treatment with a free radical scavenger, lecithinized superoxide dismutase (SOD) [11] or a neuronal NO synthase inhibitor, 7-nitroindazole [12] expanded the survival of motor neurons in wobbler mice. Therefore, multifunctional properties of ZNS may lead to neuroprotection against wobbler mouse motor neuron degeneration. Here we did not study how ZNS influences VPS54 in wobbler mice. VPS54 is a subunit of the Golgi-associated retrograde protein complex necessary for retrograde transport of vesicles to the trans-Golgi network. Axonal transport of the spinal motor neuron is impaired in wobbler mice [8]. VSP54 mutation could participate in the pathogenesis of this axonal transport damage. Multifunctional effects of ZNS may repair axonal transportation system in wobbler mice.

In conclusion, the present study indicated that ZNS had therapeutic effects on denervation muscle atrophy, motor neuron degeneration and astrocyte proliferation in wobbler mice. For clinical trials of ALS patients, further experimental studies are needed to examine whether ZNS modifies TDP43 in wobbler mice and also whether this drug can protect against motor neuron degeneration in other animal models, such as mutant SOD 1-transgenic mice.

\section{Acknowledgement}

We gratefully thank Professor Yasuo Iwasaki, Department of Neurology, Toho University Omori Medical Center, Tokyo, Japan for critical suggestion and support.

\section{Conflicts of Interest}

All authors report no conflicts of interest.

\section{References}

1. Rock DM, Macdonald RL, Taylor CP. Blockade of sustained repetitive action potentials in cultured spinal cord neurons by zonisamide (AD 810, CI 912), a novel anticonvulsant. Epilepsy Res. 1989;3(2):138-143.

2. Schauf CL. Zonisamide enhances slow sodium inactivation in Myxicola. Brain Res. 1987;413(1):185-188.

3. Suzuki S, Kawakami K, Nishimura S, Watanabe Y, Yagi K, Seino M, Miyamoto K. Zonisamide blocks T-type calcium channel in cultured neurons of rat cerebral cortex. Epilepsy Res. 1992;12(1):21-27.

4. Hayakawa T, Higuchi $Y$, Nigami $H$, Hattori $H$. Zonisamide reduces hypoxic-ischemic brain damage in neonatal rats irrespective of its anticonvulsive effect. Eur J Pharmacol. 1994;257(1-2):131-136.

5. Asanuma M, Miyazaki I, Diaz-Corrales FJ, Miyoshi K, Ogawa N, Murata M. Preventing effects of a novel antiparkinsonian agent zonisamide on dopamine quinone formation. Neurosci Res. 2008;60(1):106-113.

6. Yokoyama H, Yano R, Kuroiwa H, Tsukada T, Uchida H, Kato H, Kasahara J, et al. Therapeutic effect of a 
novel anti-parkinsonian agent zonisamide against MPTP (1-methyl-4-phenyl-1,2,3,6-tetrahydropyridine) neurotoxicity in mice. Metab Brain Dis. 2010;25(2):135143.

7. Rosler TW, Arias-Carrion O, Hoglinger GU. Zonisamide: aspects in neuroprotection. Exp Neurol. 2010;224(2):336-339.

8. Mitsumoto H, Bradley WG. Murine motor neuron disease (the wobbler mouse): degeneration and regeneration of the lower motor neuron. Brain. 1982;105 (Pt 4):811-834.

9. Schmitt-John T, Drepper C, Mussmann A, Hahn P, Kuhlmann M, Thiel C, Hafner M, et al. Mutation of Vps54 causes motor neuron disease and defective spermiogenesis in the wobbler mouse. Nat Genet. 2005;37(11):12131215.

10. Mitsumoto H, Ikeda K, Klinkosz B, Cedarbaum JM, Wong V, Lindsay RM. Arrest of motor neuron disease in wobbler mice cotreated with CNTF and BDNF. Science. 1994;265(5175):1107-1110.

11. Ikeda K, Kinoshita M, Iwasaki Y, Tagaya N, Shiojima T. Lecithinized superoxide dismutase retards wobbler mouse motoneuron disease. Neuromuscul Disord. 1995;5(5):383-390.

12. Ikeda K, Iwasaki Y, Kinoshita M. Neuronal nitric oxide synthase inhibitor, 7-nitroindazole, delays motor dysfunction and spinal motoneuron degeneration in the wobbler mouse. J Neurol Sci. 1998;160(1):9-15.

13. Iwamoto K, Yoshii Y, Ikeda K. Atorvastatin treatment attenuates motor neuron degeneration in wobbler mice. Amyotroph Lateral Scler. 2009;10(5-6):405-409.

14. Dennis JS, Citron BA. Wobbler mice modeling motor neuron disease display elevated transactive response DNA binding protein. Neuroscience. 2009;158(2):745750 .

15. Hashimoto Y, Suemaru K, Yamamoto T, Kawakami K, Araki H, Gomita Y. Effect of immobilization stress on anticonvulsant actions and pharmacokinetics of zonisamide in mice. Pharmacol Biochem Behav. 2001;68(1):7-12.

16. Hashimoto Y, Araki H, Futagami K, Kawasaki H, Gomita Y. Effects of valproate, phenytoin, and zonisamide on clonic and tonic seizures induced by acute and repeated exposure of mice to flurothyl. Physiol Behav. 2003;78(3):465-469.

17. Owen AJ, Ijaz S, Miyashita H, Wishart T, Howlett W, Shuaib A. Zonisamide as a neuroprotective agent in an adult gerbil model of global forebrain ischemia: a histological, in vivo microdialysis and behavioral study. Brain Res. 1997;770(1-2):115-122.

18. Asanuma M, Miyazaki I, Diaz-Corrales FJ, Kimoto N, Kikkawa Y, Takeshima M, Miyoshi K, et al. Neuroprotective effects of zonisamide target astrocyte. Ann Neurol. 2010;67(2):239-249.

19. Choudhury ME, Moritoyo T, Kubo M, Kyaw WT, Yabe H, Nishikawa N, Nagai M, et al. Zonisamide-induced long-lasting recovery of dopaminergic neurons from MPTP-toxicity. Brain Res. 2011;1384:170-178.

20. Mennini T, Bendotti C. Excitotoxicity in amyotrophic lateral sclerosis: selective vulnerability of motor neurons. In: Ferrarese, C., Beal, M.F. (Eds.), Excitotoxicity in Neurological Diseases: New Therapeutic Challenge. Kluwer Academic Publishers 2004, pp. 217-227.

21. Laage S, Zobel G, Jockusch H. Astrocyte overgrowth in the brain stem and spinal cord of mice affected by spinal atrophy, wobbler. Dev Neurosci. 1988;10(3):190-198.

22. Hantaz-Ambroise D, Blondet B, Murawsky M, Rieger F. Abnormal astrocyte differentiation and defective cellular interactions in wobbler mouse spinal cord. J Neurocytol. 1994;23(3):179-192.

23. Diana V, Ottolina A, Botti F, Fumagalli E, Calcagno E, De Paola M, Cagnotto A, et al. Neural precursor-derived astrocytes of wobbler mice induce apoptotic death of motor neurons through reduced glutamate uptake. Exp Neurol. 2010;225(1):163-172.

24. Das A, McDowell M, O’Dell CM, Busch ME, Smith JA, Ray SK, Banik NL. Post-treatment with voltage-gated $\mathrm{Na}(+)$ channel blocker attenuates kainic acid-induced apoptosis in rat primary hippocampal neurons. Neurochem Res. 2010;35(12):2175-2183.

25. Mori A, Noda Y, Packer L. The anticonvulsant zonisamide scavenges free radicals. Epilepsy Res. 1998;30(2):153-158. 